\title{
Analysis of Trajectory Flexibility Preservation Impact on Traffic Complexity
}

\author{
Husni R. Idris ${ }^{*}$, Nola Shen $^{\dagger}$ and Tarek El-Wakil ${ }^{\ddagger}$ \\ L3-Communications, Billerica, MA, 01821 \\ David J. Wing ${ }^{\S}$ \\ NASA Langley Research Center, Hampton, VA, 23681
}

\begin{abstract}
The growing demand for air travel is increasing the need for mitigation of air traffic congestion and complexity problems, which are already at high levels. At the same time new information and automation technologies are enabling the distribution of tasks and decisions from the service providers to the users of the air traffic system, with potential capacity and cost benefits. This distribution of tasks and decisions raises the concern that independent user actions will decrease the predictability and increase the complexity of the traffic system, hence inhibiting and possibly reversing any potential benefits. In answer to this concern, the authors proposed the introduction of decision-making metrics for preserving user trajectory flexibility. The hypothesis is that such metrics will make user actions naturally mitigate traffic complexity. In this paper, the impact of using these metrics on traffic complexity is investigated. The scenarios analyzed include aircraft in en route airspace with each aircraft meeting a required time of arrival in a one-hour time horizon while mitigating the risk of loss of separation with the other aircraft, thus preserving its trajectory flexibility. The experiments showed promising results in that the individual trajectory flexibility preservation induced self-separation and self-organization effects in the overall traffic situation. The effects were quantified using traffic complexity metrics, namely dynamic density indicators, which indicated that using the flexibility metrics reduced aircraft density and the potential of loss of separation.
\end{abstract}

\section{Nomenclature}

\begin{tabular}{ll} 
ADP & Adaptability \\
RBT & Robustness \\
RTA & Required Time of Arrival \\
$(t, x, y)$ & (time, $x$-location, $y$-location) \\
$V$ & Ground speed \\
$\mathrm{h}_{\mathrm{i}}, \mathrm{h}_{\min }, \mathrm{h}_{\max }$ & Heading with its maximum and minimum values \\
Traj & Trajectory \\
$\mathrm{P}_{\mathrm{i}}$ & Probability of trajectory instance traj \\
\hline & \\
\hline incipal Research Engineer, 300 Concord Road, Suite 400, AIAA Member \\
Research Analyst, 300 Concord Road, Suite 400, AIAA Member \\
Poftware Engineer, 300 Concord Road, Suite 400, AIAA Member \\
Principal ATM Research Engineer, Mail Stop 152
\end{tabular}




$\begin{array}{ll}P_{c} & \text { Probability of constraint situation } c \\ P_{f} & \text { Probability of feasibility } \\ P_{f, c} & \text { Probability of feasibility in constraint situation } c \\ f(t, x, y) & \text { Number of feasible trajectories from }(t, x, y) \text { to destination } \\ f_{c}(t, x, y) & \text { Number of feasible trajectories from }(t, x, y) \text { to destination in situation } c \\ i(t, x, y) & \text { Number of infeasible trajectories from }(t, x, y) \text { to destination } \\ \mathrm{i}_{c}(t, x, y) & \text { Number of infeasible trajectories from (t,x,y) to destination in situation } c \\ N(t, x, y) & \text { Number of all trajectories from }(t, x, y) \text { to destination } \\ g(x, y) \text { or } g_{k}(x, y) & \text { Number of trajectories from } k=(t, x, y) \text { to next time step } \\ \varepsilon & \text { Duration between time increments } \\ \Delta T & \text { Time horizon for conflict detection }\end{array}$

\section{Introduction}

Tn order to handle the expected increase in air traffic, the Next Generation Air Transportation System (NextGen) 1 will introduce key transformations in Air Traffic Management (ATM). ${ }^{1}$ Three examples are: increasing information sharing through net-enabled information access; making access to National Airspace System (NAS) resources dependent on aircraft equipage; and aircraft trajectory-based operations enabled by aircraft ability to precisely follow customized four dimensional (4D) trajectories. ${ }^{1}$ These capabilities enable shifting the ATM system towards a distributed architecture. ${ }^{2}$ For example, NextGen is investigating delegating more responsibility for traffic separation to the pilot ${ }^{2,3}$ and delegating more responsibility to airline operation centers for traffic flow management ${ }^{3,4}$. Enabling the gains of distributed decision making depends on the ability of distributed actions, by airborne and ground-based agents, to maintain safety and efficiency at acceptable levels.

Research on distributed ATM has focused, in part, on the distribution of separation responsibility between pilots and controllers. ${ }^{5,6,7,8,9}$ Neglecting to regulate traffic beyond the separation assurance time horizon may cause complex traffic situations, which may be difficult to control whether by ground-based or by aircraft-based agents, leading to compromised safety. Therefore, reducing or preventing such situations is a prerequisite to enabling manageable separation assurance and safety. In order to mitigate traffic complexity, ground and airborne systems may benefit from preserving trajectory flexibility. Trajectory flexibility preservation enables an aircraft to plan its trajectory such that it preserves a requisite level of maneuvering flexibility in order to accommodate later disturbances caused, for example, by other traffic and weather activity. The hypothesis is that if each aircraft preserves its own trajectory flexibility, using an air-based or ground-based system, acceptable traffic complexity will naturally be achieved. As discussed in Idris et al., although flexibility preservation does not explicitly coordinate between aircraft, it assists each by reducing the risk of conflict due to the potential behavior of the surrounding traffic, thus resulting in implicit coordination. ${ }^{10,11}$ This function offers a trajectory-oriented approach to managing traffic complexity, by explicitly planning aircraft trajectories, such that their contribution to complexity is minimized. This is contrasted with airspace-oriented approaches that aim to ensure that airspace characteristics (such as sector size and route patterns) and traffic characteristics (such as aircraft density) are designed to dynamically limit traffic complexity.

Flexibility preservation complements separation assurance both within the conflict resolution horizon and beyond it to an extended flexibility planning horizon. Within the conflict resolution horizon, flexibility aids in selecting conflict resolution solutions that afford the aircraft more flexibility, for example, to adapt to potential intruder behavior. Beyond the conflict resolution horizon, which is the focus of this paper, flexibility preservation plans the aircraft trajectory to minimize its exposure to disturbances such as weather cells and dense traffic. Fig. 1 depicts an example. In its left portion each aircraft, while planning its trajectory between weather cells, questions whether it should modify its trajectory to increase flexibility. If the aircraft proceed along their depicted headings, a complex traffic situation arises causing excessive congestion and a high potential conflict rate. On the other hand, the right portion displays a structured traffic pattern that would result if each aircraft maneuvered to increase its own flexibility. 


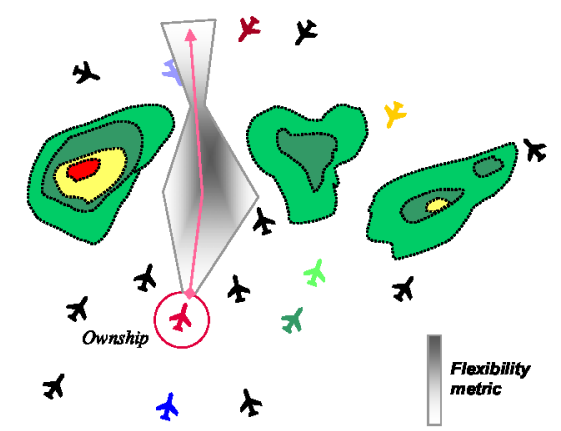

Airborne flexibility function will question: Do I have enough flexibility to safely proceed? Can I modify my trajectory to increase my flexibility? Do I need to avoid this airspace entirely and replan?
Trajectories Designed to Preserve Flexibility

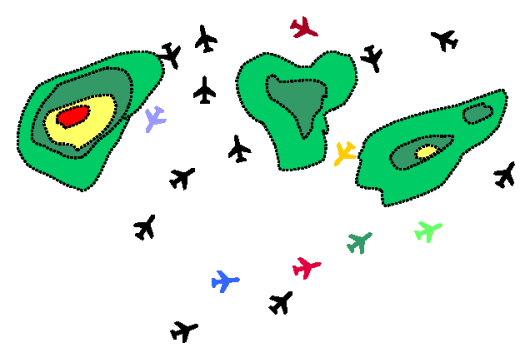

Hypothesis:

If all aircraft apply flexibility preservation function, complexity automatically will be reduced

Figure 1 Trajectory flexibility preservation avoiding weather cells and congestion

To test this hypothesis, trajectory flexibility metrics have been defined in previous work to represent robustness and adaptability to the risk of violating separation, airspace hazards, and traffic flow management constraints. ${ }^{11-13}$ The impact of trajectory planning by preserving these metrics is analyzed on traffic complexity metrics selected from the literature. Many approaches have been documented to define and measure traffic complexity, most often as a function of controller workload. For example, dynamic density measures traffic complexity using a number of factors. These factors are primarily based on airspace geometry such as aircraft density, sector geometry, traffic flow structure, and mix of aircraft types and performance characteristics. ${ }^{14-17}$ Other efforts emphasized cognitive elements of complexity, in particular the controller use of standard flows, grouping of traffic, and merge points. ${ }^{18}$ Some metrics have been proposed that are independent of the airspace structure and controller perspective. For example, Delahaye et al. ${ }^{19}$ introduced complexity metrics based on traffic organization or disorder based on Lyapunov exponents. In a previous paper ${ }^{20}$, the impact of preserving trajectory flexibility was analyzed on the Lyapunovexponent-based metrics. In this paper the impact on dynamic density metrics is analyzed.

Two scenarios are analyzed in two-dimensional en route airspace, where each aircraft must meet a required time of arrival (RTA) in a one-hour time horizon using speed and heading degrees of freedom. Simultaneously, each aircraft preserves its trajectory flexibility, using the defined metrics, to mitigate the risk of loss of separation with hazards and the other aircraft. The effects are quantified using traffic complexity metrics based on flow pattern consistency and dynamic density. The experiments showed promising results in terms of mitigating complexity as measured by these metrics.

\section{Metrics}

Metrics that represent trajectory flexibility have been developed and reported in previous papers. ${ }^{12,13}$ They are summarized briefly in this section. To test the hypothesis, traffic complexity metric were also selected. These metrics are also briefly described in this section.

\section{A. Trajectory Flexibility Metrics}

The notion of "trajectory flexibility" was defined in Idris et al. ${ }^{11}$ as the ability of the trajectory (and hence the aircraft following the trajectory) to abide by all constraints imposed on it while mitigating its exposure to risks that cause violation of these constraints. The constraints intend to achieve ATM and aircraft objectives and include heading limits, RTAs, and separation minima. They define the trajectory solution space. Risk of constraint violation is represented by disturbances that cause the aircraft trajectory to violate or potentially violate constraints. Disturbances were classified in Idris et al. ${ }^{11-13}$ into state disturbances that result in aircraft state deviation along its trajectory or constraint disturbances such as new constraints or modifications of currently imposed or known potential constraints.

Two trajectory characteristics relevant to measuring this notion of flexibility have been identified: robustness and adaptability. ${ }^{11}$ Metrics have been proposed for robustness and adaptability based on estimating the number of feasible trajectories available to the aircraft to accommodate disturbances. ${ }^{12,13}$ They are summarized here briefly. In order to support these definitions and estimation methods, the aircraft is assumed to follow segments of discrete time length, where instantaneous heading and speed changes can only occur at discrete instances in time that are $\varepsilon$ apart. 
Also, heading $h$ and speed $V$ take discrete values between $h_{\min }$ and $h_{\max }$ and between $V_{\min }$ and $V_{\max }$ and are constant along each segment. (Altitude is not considered in this paper.) In addition to simplifying the estimation method, these assumptions are reasonable from an operational point of view considering the intended application of the trajectory flexibility metrics. Namely, the metrics are intended for relative comparison of trajectories over a long time horizon suitable for strategic planning (typical of traffic flow management planning horizon) as opposed to tactical maneuvering (where the dynamics of the speed and heading change are relevant).

(1) Robustness is defined as the ability of the aircraft to keep its planned trajectory unchanged in response to the occurrence of disturbances, for example, no matter which trajectory or conflict instances materialize. A robustness metric $\operatorname{RBT}(\mathrm{t}, \mathrm{x}, \mathrm{y})$ is measured with the probability of feasibility $\mathrm{P}_{\mathrm{f}}($ traj) of the trajectory (traj) starting from a state $(t, x, y)$ and ending at another state such as (RTA, $\left.x_{\text {dest }}, y_{\text {dest }}\right) . P_{f}(t r a j)$ can be estimated with partial information about state and constraint disturbances, modeled with probability distributions that represent the risk of constraint violation. As an example, the constraints are modeled with $\mathbf{C}$ constraint situations $\mathbf{c}$, each with a probability $\mathbf{P}_{\mathbf{c}}$ with $\sum_{c=1: C} P_{c}=1$. Each constraint situation $c$ divides the total set of trajectories $N(t, x, y)$ into two mutually exclusive subsets: $f_{c}(t, x, y)$ the set of feasible trajectories and $i_{c}(t, x, y)$ the set of infeasible trajectories, both with respect to $c$. Then, the following formula can be derived for robustness RBT(t, $x, y)$; see Idris et al. ${ }^{12,13}$ for more details:

$$
P_{f, c}(t, x, y)=\sum_{\text {set of feasible trajectoryinstancesi } i} P\left(t_{r} j_{j}\right)=\frac{f_{c}(t, x, y)}{N(t, x, y)}, \quad R B T(t, x, y)=P_{f}(t, x, y)=\sum_{c=t: C} P_{c} \times \frac{f_{c}(t, x, y)}{f_{c}(t, x, y)+i_{c}(t, x, y)}
$$

where $P_{f, c}$ is the probability of feasibility of the trajectory traj in a constraint situation $c$, which is the ratio of the number of feasible trajectories $f_{c}$ to the total number of trajectories $N$, if the probability of each trajectory instance $P\left(\right.$ traj $\left._{j}\right)$ is equal to $1 / \mathrm{N}(t, x, y)$.

(2) Adaptability is defined as the ability of the aircraft to change its planned trajectory in response to the occurrence of a disturbance that renders the current planned trajectory infeasible. An adaptability metric ADP( $t, x$, $y$ ) is measured by the number of feasible trajectories $f(t, x, y)$ (with respect to all constraints) that are available for the aircraft to use at $(t, x, y)$ to regain feasibility. Then, given the probability distribution $\left(P_{c}\right)$ of each constraint situation $c$ of $C$, ADP may be estimated by the average over $C$ :

$$
\operatorname{ADP}(t, x, y)=f(t, x, y)=\sum_{c=1: C} P_{c} \times f_{c}(t, x, y)
$$

Adaptability decreases as the aircraft moves along a trajectory because the number of feasible trajectories decreases. The special case of robustness given by (1) (robustness to totally random state disturbances) increases over time because as the number of feasible trajectories (numerator) decreases the total number of trajectories (denominator) decreases more rapidly by both infeasible and feasible trajectories.

\section{B. Traffic Complexity Metrics}

The impact of planning trajectories using the adaptability and robustness metrics on traffic complexity was assessed using two main indicators: Consistency of a resulting flow pattern and dynamic density. Flow pattern consistency was measured by the percentage of aircraft that followed a consistent pattern. The pattern was readily apparent visually so no clustering technique was employed in the scenarios analyzed in this paper. The pattern was scenario dependent as will be described in Section IV. A number of dynamic density indicators were selected from the literature ${ }^{14-17}$, based on applicability (for example excluding indicators that are based on altitude variation and on sector geometry). The indicators were not combined in a single metric because such combination would reflect specific operational environment. Rather the indicators were measured and reported independently. The following is a brief description of each of the indicators:

(1) Traffic density is the number of cells (the whole analyzed area is divided into sixty-four cells with equal size) occupied by more than a certain number of aircrafts for each instant of time.

(2) Horizontal proximity is the inverse of the average weighted horizontal separation between all the aircraft pairs for each instant of time. For each aircraft, the weighted horizontal separation is estimated based on the distance to all the other aircrafts. Those aircrafts closer to the own aircraft are given more weight. ${ }^{16}$

(3) Variance of headings using the standard deviation of the headings of all the aircrafts within a defined area. The area is selected (as described in Section III.B and shown in Fig. 5 and 6) instead of the full area so that the aircraft movement pattern can be reflected in the metric.

(4) The number of conflicts is the number of aircraft pairs with impending conflicts in a time horizon $\Delta T$. The measure is normalized by the number of aircrafts for each time step. Both aircraft state-based and the trajectorybased conflicts are detected. 10 minutes conflict prediction time horizon was used in this analysis. For state-based conflicts, the shortest distance between the aircraft pairs is estimated based on their speed, heading and initial separation. This shortest distance is then compared with the minimum horizontal separation (a 5 nautical mile 
separation requirement was used) according to a published algorithm ${ }^{21}$. The conflict is detected for this aircraft pair if the estimated shortest distance is less than the minimum horizontal separation. The number of conflicts is also estimated according to the resulting aircraft trajectories. For each time step, the aircraft pairs with horizontal separation less than the minimal horizontal separation in the next 10 minutes is counted as impending conflict.

(5) Crossing angle is the average crossing angle of the aircraft pairs with impending conflict in $\Delta T$. The crossing angle is the acute angle between the paths of two converging aircraft predicted to be in conflict using the state projection.

(6) Time to conflict is the inverse of average minimum time to the conflict for all the aircrafts with impending conflicts in $\Delta T$. For aircraft state-based conflicts, the time to conflict is the time to the closest approach point. While for the trajectory-based conflicts, the time to conflict is to the time when the aircraft pair first lost the minimum separation.

\section{Trajectory Generation Algorithm}

A dynamic programming algorithm was used to generate an aircraft trajectory using the robustness and adaptability metrics. Because the intention of this analysis is to test a hypothesis rather than a real-time application, the dynamic programming approach was selected due to its simple formulation and despite its numerical and storage limitations. First the trajectory solution space is built as a tree of discrete states connected according to reachability by the allowable discrete speed and heading values over the discrete time increments. Second, the robustness and adaptability metrics are estimated at each state. Third and finally, a back-propagation algorithm computes a cost function and builds a trajectory that optimizes the cost function.

\section{A. Flexibility Metric Estimation}

Under the assumptions of discrete time and degrees of freedom, the number of trajectories was estimated using a convolution and filtering technique. ${ }^{11}$ Fig. 2 demonstrates this method for calculating $f_{c}(t, x, y)$ from any point $(t, x, y)$ to a destination specified by a point (RTA, $x_{\text {dest }}, y_{\text {dest }}$ ) and a tolerance circle around it in the $\mathrm{x}-\mathrm{y}$ plane, in a constraint situation $\mathrm{c}$ that includes an instance of a potential conflict. The 3-dimensional space is discretized into time steps $\varepsilon$ apart, where in each time step the $x-y$ plane is discretized into square cells. The function $f_{c}(t, x, y)$ is estimated for each cell. Assume the function $f_{c}\left(t_{j}, x, y\right)$ at time $t_{j}$ is known. The function $f_{c}\left(t_{j-1}, x, y\right)$ at the previous time step $t_{j-1}$ can be obtained by convoluting $\mathrm{f}_{\mathrm{c}}\left(\mathrm{t}_{\mathrm{j}}, \mathrm{x}, \mathrm{y}\right)$ and the

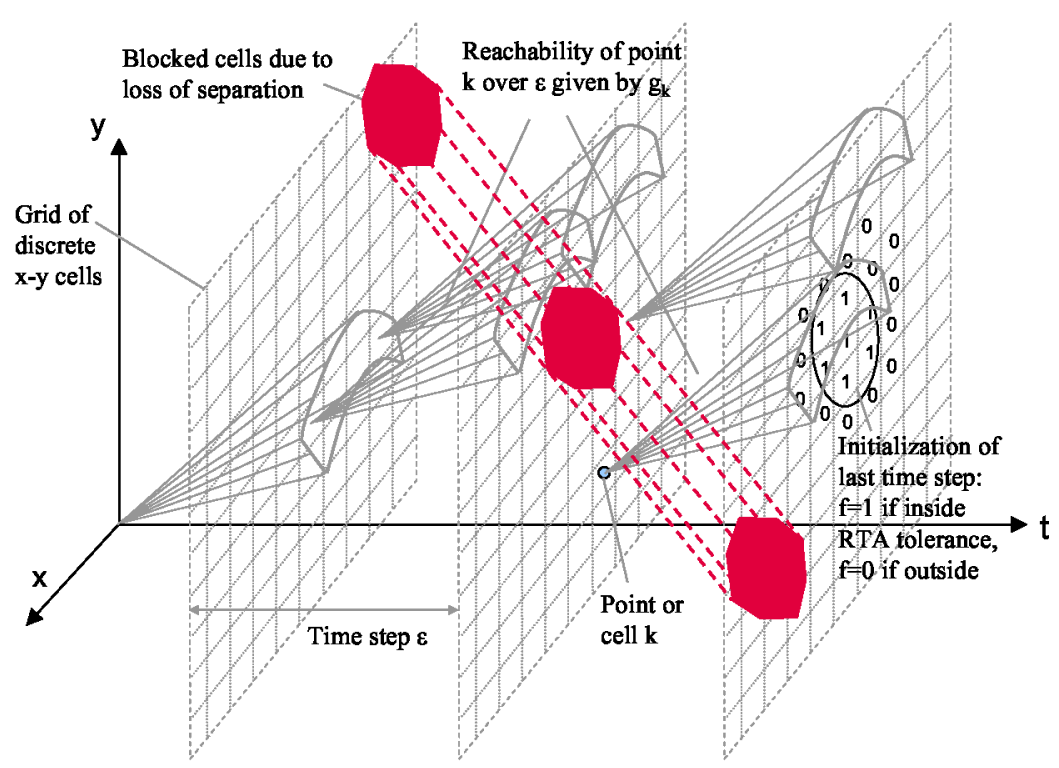

Figure 2. Discrete estimation of number of feasible trajectories function $\mathrm{g}_{\mathrm{k}}(\mathrm{x}, \mathrm{y})$, which represents the number of trajectories that reach from a point $\mathrm{k}=\left(\mathrm{t}_{\mathrm{j}-1}, \mathrm{x}(\mathrm{k}), \mathrm{y}(\mathrm{k})\right)$ at time step $t_{j-1}$ to the next time step $t_{j}$. The function $g_{k}(x, y)$ is shown as a conical shell in Fig. 2. However, if the point $k$ is infeasible (for example due to loss of separation) then $f_{c}\left(t_{j-1}, x(k), y(k)\right)=0$. This requires a filtering step before each convolution operation to zero out the values at infeasible states. Substituting a dummy variable $\tau$ to denote sliding the point $k$ in the $x-y$ plane, the function $f_{c}\left(t_{j-1}, x, y\right)$ is given by the following equation, representing convolution and filtering for infeasibility:

$$
\begin{array}{ll}
\mathrm{f}_{\mathrm{c}}\left(\mathrm{t}_{\mathrm{j}-1}, \mathrm{x}, \mathrm{y}\right)=\sum_{\lambda} \sum_{\tau} \mathrm{f}_{\mathrm{c}}\left(\mathrm{t}_{\mathrm{j}}, \tau, \lambda\right) \mathrm{g}(\mathrm{x}-\tau, \mathrm{y}-\lambda) & \text { if feasible } \\
\mathrm{f}_{\mathrm{c}}\left(\mathrm{t}_{\mathrm{j}-1}, \mathrm{x}, \mathrm{y}\right)=0 & \text { if infeasible }
\end{array}
$$

This operation is applied starting from the destination step $t=R T A$ and proceeding backwards to the current state. The destination time step is initialized by setting $\mathrm{f}_{\mathrm{c}}(\mathrm{RTA}, \mathrm{x}, \mathrm{y})=1$ at the feasible states and zero elsewhere as shown in Fig. 2. To compute the total number of trajectories, $N(t, x, y)$ used in the denominator of the robustness 
metric RBT, certain constraints are excluded from the filtering process (namely the constraints with respect to which robustness is computed). In this paper robustness only to loss of separation with traffic and hazards is considered. Therefore, the numerator filtering was applied to all cells that lead to separation loss as well as cells that lead to violating speed and heading limits or violating the RTA constraint. On the other hand, filtering ignored loss of separation but was applied to the RTA and heading and speed limit constraints for calculating the denominator.

Separation zones were modeled as circles with given radii surrounding each intruder aircraft trajectory. Because a trajectory consists of discrete segments, each with constant speed and heading, the circle moves with constant speed and heading for the duration of each segment. In each segment, the circle is enclosed with eight tangent planes, each two opposing tangents resulting from a combination of heading and speed limits of the ownship aircraft relative to the intruder (There are four such combinations). A cell loses (or is imminent to lose) separation if it falls on the inside of all eight planes, within the time duration of the segment. Hazards are similarly modeled as circles with zero speed. Under probabilistic models of disturbances, the estimation process is repeated for each constraint situation $c$. Then, the estimates $f_{c}(t, x, y)$ are averaged over all situations $C$ to obtain the adaptability or robustness metrics (1) and (2). Examples of ADP and RBT are shown in Fig. 3 and 4 for an analysis case.

\section{B. Cost Function and Trajectory Building}

Using recursive back-propagation, starting from the final time step, the minimum cost of proceeding from each cell to the destination is computed and stored. This minimum cost $Q(t, x(k), y(k))$ for each cell $k$ is computed by minimizing, over its reachable cells given by $\left\{(t+1, x, y): g_{k}(t+1, x, y)=1\right\}$ in the next time step $t+1$, the sum of the minimum cost $\mathrm{Q}(\mathrm{t}+1, \mathrm{x}, \mathrm{y})$ already computed for each of the reachable cells $(t+1, \mathrm{x}, \mathrm{y})$ plus the cost of proceeding from $\mathrm{k}$ to that cell, given for short by $\mathrm{q}(\mathrm{k} \rightarrow(\mathrm{t}+1, \mathrm{x}, \mathrm{y}))$. A generic formula is:

$$
\mathrm{Q}(\mathrm{t}, \mathrm{x}(\mathrm{k}), \mathrm{y}(\mathrm{k}))=\operatorname{Min}_{\mathrm{x}, \mathrm{y}: \mathrm{g}_{\mathrm{k}}(\mathrm{x}, \mathrm{y})=1}\{\mathrm{Q}(\mathrm{t}+1, \mathrm{x}, \mathrm{y})+\mathrm{q}(\mathrm{k} \rightarrow(\mathrm{t}+1, \mathrm{x}, \mathrm{y})\}
$$

Four functions for the local cost $q$ were used in the experiments reported in this paper. A function representing minimizing path length was used as a baseline. Then, functions representing maximizing adaptability, maximizing robustness, and maximizing both combined with minimizing path length:

$$
\begin{aligned}
& \mathrm{q}(\mathrm{k} \rightarrow(\mathrm{t}+1, \mathrm{x}, \mathrm{y}))=\operatorname{distance}(\mathrm{k} \rightarrow(\mathrm{t}+1, \mathrm{x}, \mathrm{y}))=\text { dist } \\
& \mathrm{q}(\mathrm{k} \rightarrow(\mathrm{t}+1, \mathrm{x}, \mathrm{y}))=-\operatorname{ADP}(\mathrm{k}) \\
& \mathrm{q}(\mathrm{k} \rightarrow(\mathrm{t}+1, \mathrm{x}, \mathrm{y}))=-\operatorname{RBT}(\mathrm{k}) \\
& \mathrm{q}(\mathrm{k} \rightarrow(\mathrm{t}+1, \mathrm{x}, \mathrm{y}))=-\operatorname{ADP}(\mathrm{k})-\mathrm{a}^{\mathrm{T}} \mathrm{RBT}(\mathrm{k})+\mathrm{b}^{\mathrm{T}} \text { dist }
\end{aligned}
$$

where $a$ and $b$ are weights that trade robustness and distance, respectively, with adaptability. They are raised to the power of time $\mathrm{T}$ (measured from the end) to account for the exponential growth of ADP. After storing the optimal costs for each cell, a forward loop builds a trajectory by tracing the optimal cells starting from the initial state. Any ties were broken randomly.

\section{Complexity Impact Analysis}

The estimation technique and trajectory optimization algorithm were implemented in a MATLAB tool. The resulting trajectories were analyzed using the traffic complexity metrics described in Section III.B. First the scenarios reported in this paper are described. Then, observations are made on the impact of trajectory planning, using the four cost functions (4) through (7), on traffic complexity.

\section{A. Analysis Scenario}

The first of the two scenarios analyzed in this paper consists of a line of weather cells leaving two holes for which two flows of traffic compete. The two traffic flows travel in opposite directions: one starts at $x=0, y=-120$ nautical miles and heads towards $x=0, y=80$ nautical miles. The other flow starts at $x=0, y=120$ and ends at $x=$ $0, y=-80$ nautical miles. Five weather hazard cells are modeled as circles with radius of 20 nautical miles, and located at $x=0$ and $y=\{0, \pm 70, \pm 120$ nautical miles $\}$. See Fig. 5 in Section B with the results. The geometry of the hazards and of the traffic start and end positions is selected to provide symmetry, such that the path length alone is not a differentiator between selecting among the two holes. This ensures highlighting the impact of the robustness and adaptability metrics compared to shortest path. Each traffic flow is generated with random entry times separated by intervals between 5 and 7 minutes. All aircraft are limited to headings of \pm 60 degrees relative to the centerline connecting the start and end positions, with 10-degree increments. They are also limited to a speed between 240 and 360 knots with 10-knot increments. Each aircraft is assigned an RTA at the destination that forces the aircraft to path 
stretch to meet the RTA. This was ensured by setting the RTA up to 10 minutes above the travel time at minimum speed along a straight path. The RTA is met exactly with no tolerance at the destination point allowed.

The second scenario consists of a weather cell that causes four traffic flows crossing at right angles to go around the weather cell in a roundabout. The weather cell is modeled as a circle with radius of 30 nautical miles located at $(x=0, y=0)$. The four traffic flows originate at $(x=0, y=-120),(x=0, y=120),(x=-120, y=0)$ and $(x=120$, $y=0)$. They end respectively at $(x=0, y=80),(x=0, y=-80),(x=80, y=0)$ and $(x=-80, y=0)$. All units are in nautical miles. Eight other hazard circles are added at the corners as shown in Fig. 6 (discussed in Section B) to increase the traffic interaction around the hazard located in the center. The speed and heading limits and increments are the same as in the first scenario. The entry times for each flow ranged between 6 and 8 minutes.

In both scenarios, each aircraft plans a trajectory to meet the RTA (using speed reduction and path stretching), optimizing the four cost functions (4) through (7). Time increments of 2 minutes and square $x-y$ cells of 2 nautical miles are used in the estimation of the number of trajectories. The first aircraft does not encounter any traffic as it plans its trajectory. Then, each following aircraft plans its trajectory assuming knowledge of the trajectories of all preceding aircraft. These trajectories are surrounded by separation zones that, in addition to the weather hazards, are avoided by the aircraft. Hazards and separation zones reduce the number of feasible trajectories. Therefore, earlier aircraft are given priority while each later aircraft encounters exceedingly more traffic. No dynamic trajectory modification is considered in the experiments run for this analysis. Each aircraft generates one trajectory upon its entry and maintains this trajectory throughout. Also the experiment runs considered only deterministic aircraft behavior. One trajectory is considered for each aircraft with probability of one. However, the separation requirement around each aircraft was set to 10 nautical miles (instead of the required 5 nautical miles) in order to capture the higher uncertainty in the rather long time horizon of these experiments. Fig. 3 shows an example of the adaptability metric (ADP) at one time step of the solution space, for an aircraft that encounters the hazards of the first scenario. Color shades are used to depict the log of the number of feasible trajectories. Fig. 4 shows an example of the robustness metric (RBT) at one time step of the solution space using color shades. Note that adaptability is highest near the center of the solution space around the central hazard, while robustness is highest near the extremities of the solution space away from the central hazard. Also note that robustness here is with respect to the hazards and loss of separation only and not to the RTA constraint or the speed and heading limits. Finally it should be noted that the solution space is smaller in Fig. 4 because it is an earlier time step and that these figures are in a relative frame with respect to the aircraft (hence the hazard y-location is 120 nautical miles rather than zero.

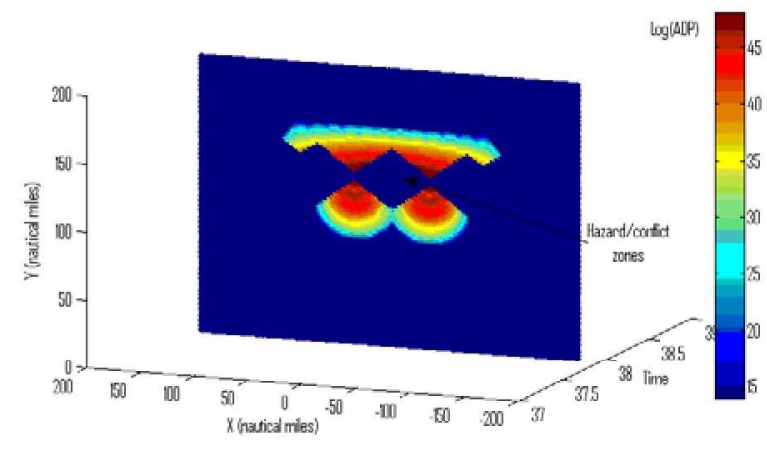

Figure 4. Example of adaptability metric map

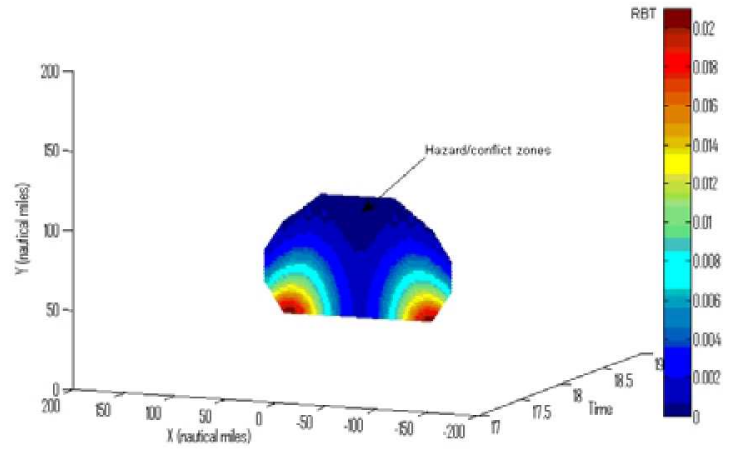

Figure 3. Example of robustness metric map

Each scenario contained 80 aircraft distributed evenly among the flows. The resulting trajectories consist of heading and speed decisions at each 2-minute increment. Finally they are analyzed for traffic complexity.

\section{B. Results and Observations}

Fig. 5 (a-e) demonstrate the resulting flow patterns in the first scenario and Fig. 6 (a-e) those in the second scenario, using full trajectories. As a baseline the shortest path cost function was run twice, once without avoiding the other traffic (cases a) and once with avoiding it (cases b). Traffic avoidance was turned off to depict current practice where conflict avoidance is only applied in a short time horizon of 10-20 minutes. Shortest-path with traffic avoidance sets another baseline for demonstrating the marginal effect of using the adaptability and robustness metrics instead. When using adaptability and robustness metrics (cases c-e) traffic is naturally avoided 


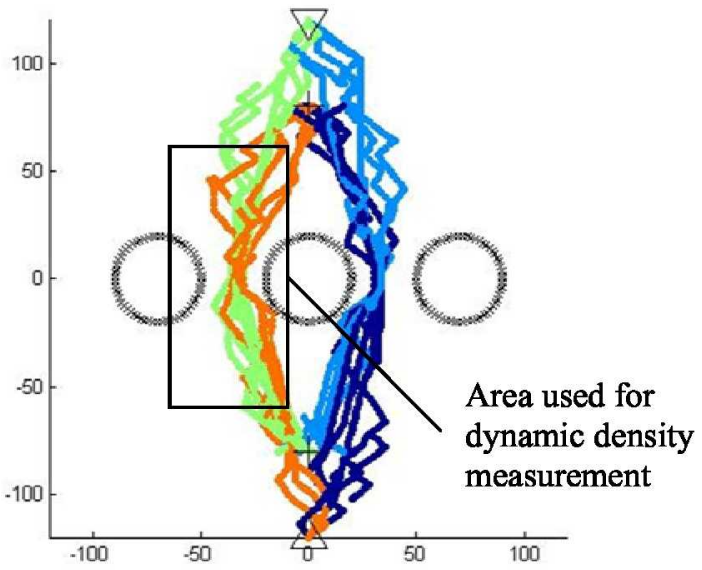

(a) Shortest path without traffic avoidance No specific pattern

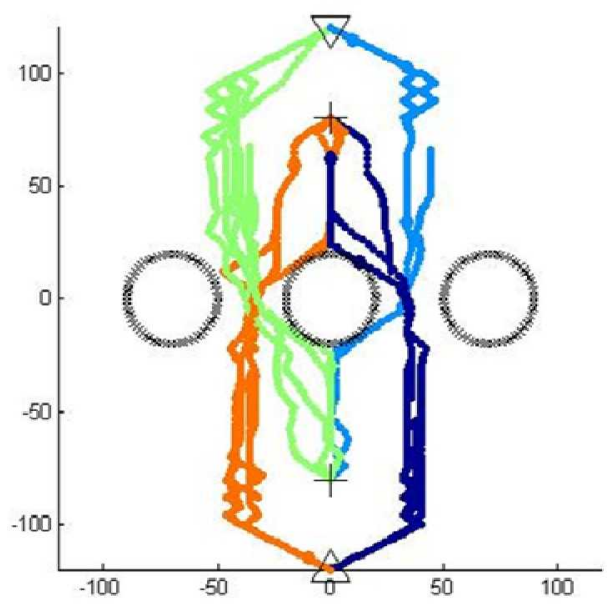

(c) Maximum adaptability only

Pattern: outer lanes before hole, centerline after hazard

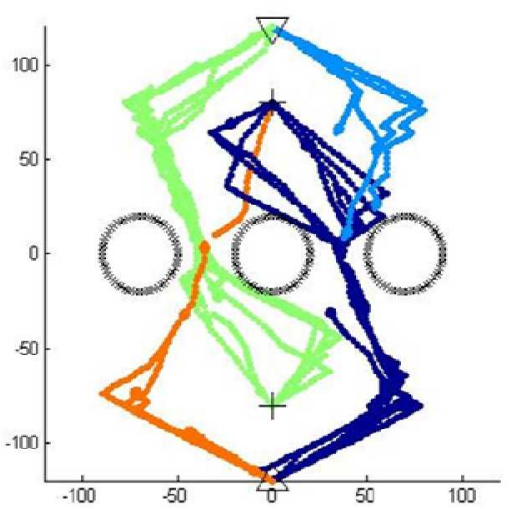

(e) Maximum adaptability and robustness, and shortest path

Pattern: Mostly northbound through right hole and southbound through left hole

Figure 5. Flow patterns in weather line scenario

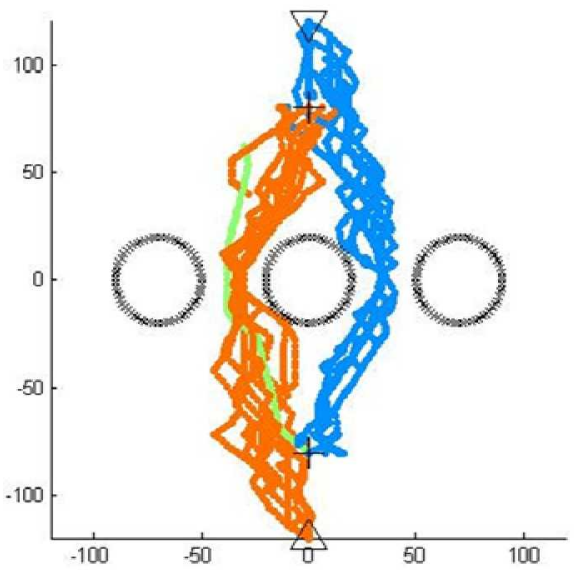

(b) Shortest path with traffic avoidance

Pattern: northbound mostly through left hole, southbound mostly through right hole

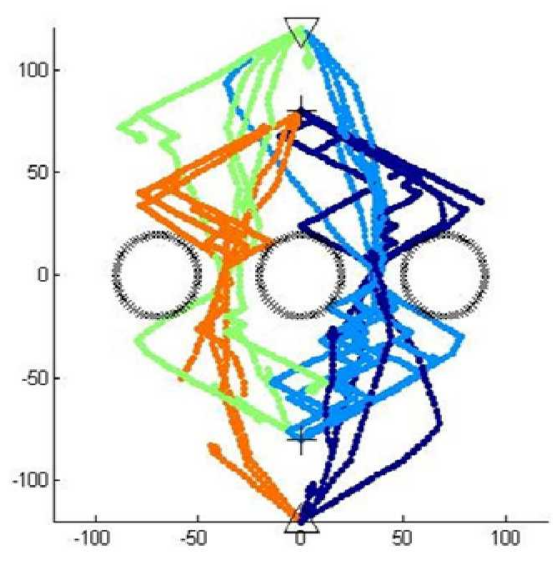

(d) Maximum robustness only

Pattern: spread out as possible

because the number of trajectories at cells that lose separation is zero. However, loss of separation is not guaranteed because of the coarse discretization of the solution space. The larger the time and space increments are the larger the chance of losing separation.

These figures demonstrate that in both scenarios, using robustness and adaptability as objectives for individual trajectory planning resulted in more structured aggregate traffic flow. Looking at the headings of the aircraft, shown by a circle at the end of the trajectories, one can see the following. In case a, which used shortest path without traffic avoidance, aircraft varied in selecting their path relative to the hazard in both scenarios resulting in closer proximity and more random flow patterns.

All the other cases resulted in a more structured traffic pattern but in a different manner: In case b of Fig. 5, which used shortest path but avoided traffic, most aircraft traveled through the holes in a uniform direction, with occasional misalignment. In case $c$ of Fig. 5, which used adaptability, 


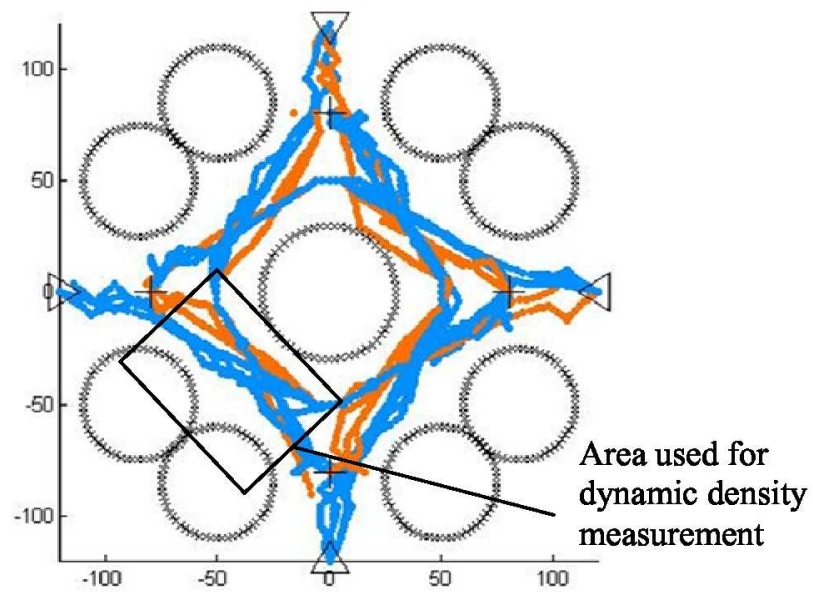

(a) Shortest path without traffic avoidance

No pattern: $60 \%$ of aircraft counterclockwise (blue)

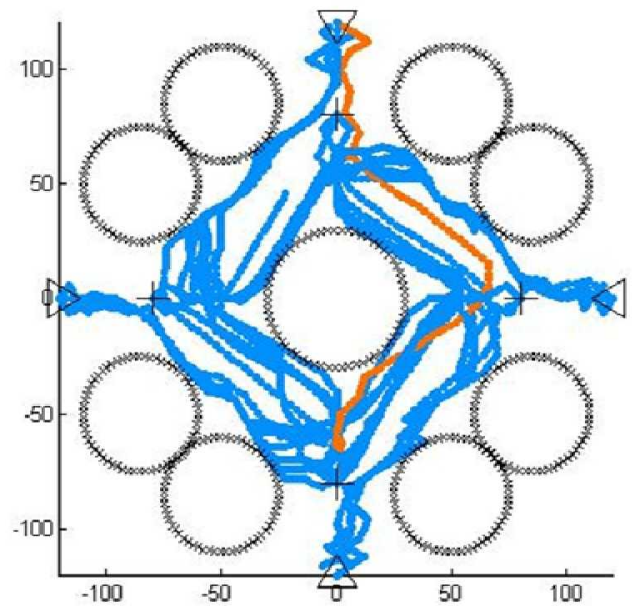

(c) Maximum adaptability only

Pattern: $97 \%$ of aircraft counterclockwise (blue)

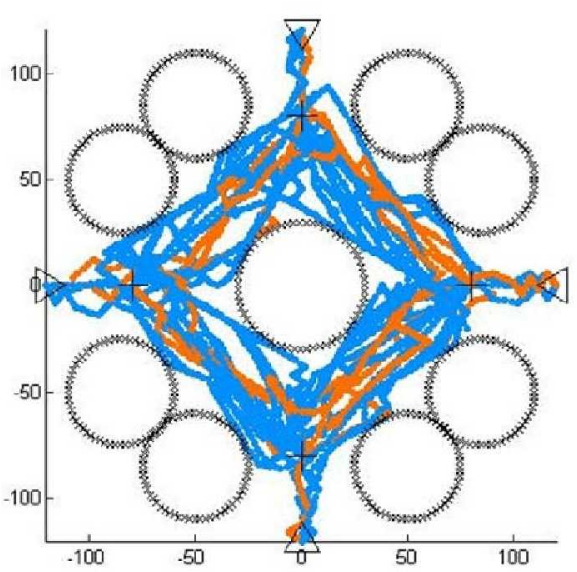

(e) Maximum adaptability and robustness, and shortest path

Pattern: $84 \%$ of aircraft counterclockwise (blue)

Figure 6. Flow patterns in roundabout scenario

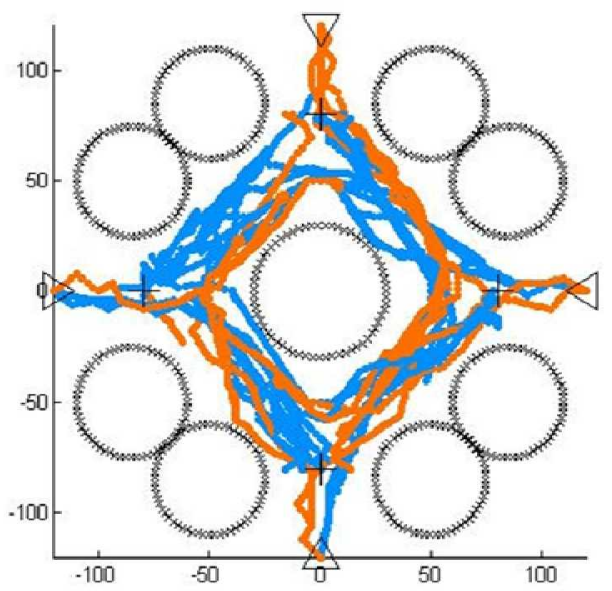

(b) Shortest path with traffic avoidance

Pattern: $68 \%$ of aircraft counterclockwise (blue)

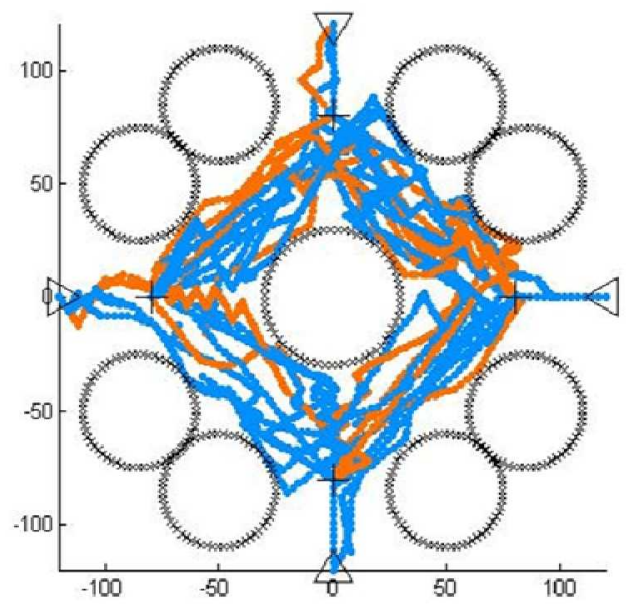

(d) Maximum robustness only

Pattern: $70 \%$ of aircraft counterclockwise (blue)

aircraft formed outer lanes before the hazard and traveled along the centerline afterwards. This pattern resulted because adaptability tended to concentrate the aircraft trajectory close to the centerline connecting the initial and final locations. This is because the number of feasible trajectories is highest near the centerline (as shown in Fig. 3 ) which caused the aircraft to hug the central hazard. The holes in this scenario were large enough to allow the aircraft to travel through them in both directions. On the other hand, robustness, which was used in case d, tended to send the aircraft away from each other and from the hazards increasing the spacing between them. This caused aircraft in case $d$ to spread out more than in cases $b$ and $c$, and to exhibit a less structured manner. Aircraft in both cases $\mathrm{c}$ and $\mathrm{d}$ separated from each other more than in cases $a$ and $b$. In case $e$, the aircraft formed a unidirectional flow through each of the holes.

In the roundabout scenario of Fig. 6, most aircraft turned around the central hazard in a uniform direction 
relative to the shortest path case a. This is indicated in the figure by the percentage of aircraft that selected the counterclockwise direction (using the darker, blue color). This percentage is higher in cases c-e (70-97 percent) than cases $a$ and $b(60-68$ percent).

The manner and degree to which the traffic self organizes depends on a number of factors. For example, the following additional observations are made:

(1) cases e of Fig. 5 and 6 combine shortest path, adaptability and robustness in the cost function (12), with $a=$ 40 and $b=5000$. These cases exhibited aspects from each of the $b, c$, and d cases: Because of robustness, aircraft spread out more. Because of adaptability, they formed a lane closer to the centerline especially after the hazard. Because of minimizing distance trajectories are smoother. The weights used in this example were not optimized and the tradeoff between these factors is a subject of further research.

(2) The density of the traffic, a function of both the arrival rate and the size of the hazards, affects the pattern. For example, the aircraft managed to go through the holes in Fig. 5 in both directions.

(3) The first aircraft in the scenario does not encounter any traffic and hence makes random decisions if there are ties between trajectories. The emerging traffic pattern depends on these early decisions. For the same reason, when traffic density declines the pattern may switch to a new one.

(4) All aircraft in these scenarios used the same objective function. This induces implicit coordination and rules and influences the emerging pattern.

(5) The shortest path case, with traffic avoidance (b) is closer to the adaptability case (c) than the robustness case (d). This is because the shortest path is close to the centerline where adaptability is high. The shortest path trajectory, however, differs from the most adaptable trajectory because it uses the minimum speed (to minimize path stretching). Therefore these trajectories were smoother and exhibited less turns. Adaptable trajectories on the other hand tended to zigzag around the centerline. Note that the jaggedness of the trajectories in all cases is an artifact of the low fidelity model used for this initial analysis to test the concept. Smoothness will be addressed and added in future research while integrating the function with a higher fidelity trajectory generator.

The resulting aircraft trajectories were also analyzed for the dynamic density indicators listed in Section II.B. The results are shown in Fig. 7 through 11, where the left side plots are for the weather-line scenario of Fig. 5 and the plots on the right side are for the roundabout scenario of Fig 6.

Figure 7 shows the aircraft density over a grid of sixty four square cells into which the full areas of the scenarios in Fig. 5 and 6 were divided. The number of cells with more than 3 aircraft over the duration shows that the cases (c) and (e) where aircrafts use adaptability have less high density cells than all the other cases for the weather line scenario of Fig. 5 (left plot). Case (a) with shortest path without traffic avoidance exhibits more cells with high traffic density. While in the right-side plot, for the roundabout scenario of Fig. 6, all the cases that avoided traffic (cases b-e) showed similar density, probably because this scenario is more constraining in terms of available airspace.
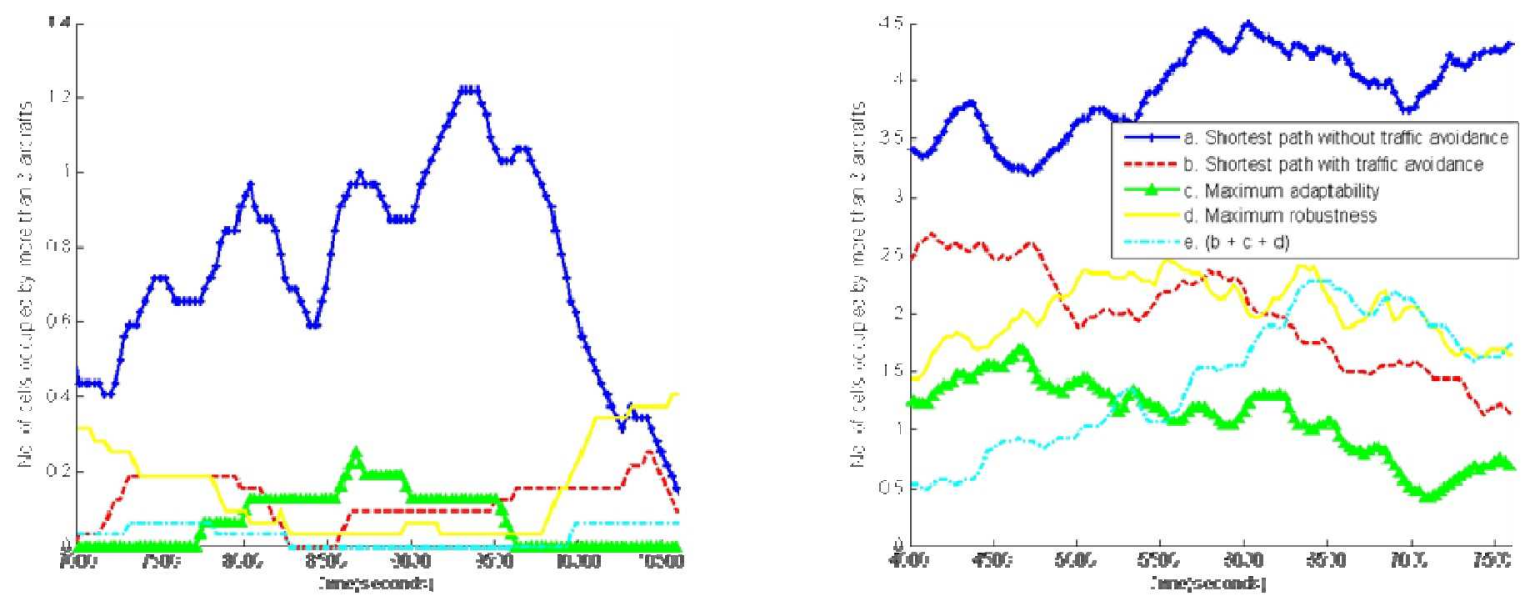

Figure 7. Aircraft density analysis 
Figure 8 compares the cases in terms of proximity. For both scenarios, case (a) where aircraft used shortest path without avoidance consistently shows the highest proximity measure. All cases (b-e) where aircrafts avoided traffic exhibit similar proximity.
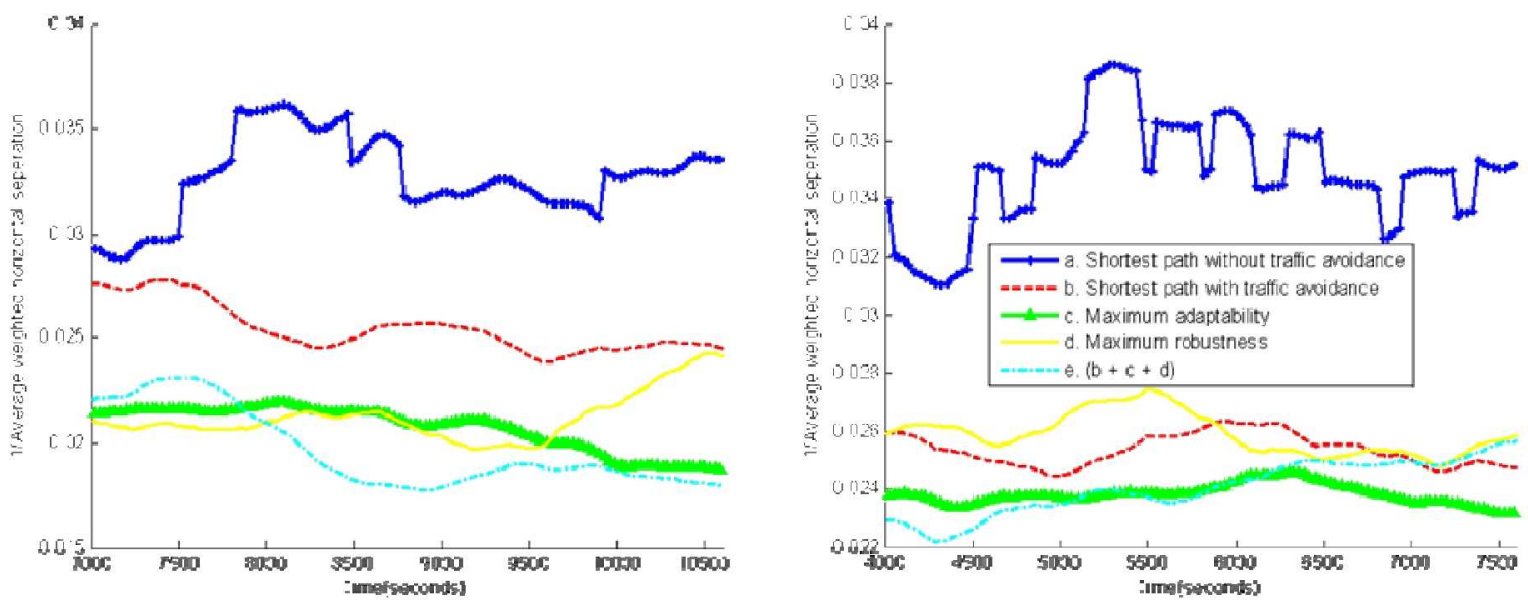

Figure 8. Proximity analysis

Figure 9 compares the average heading within bounded areas. The areas were selected to make sure that each reflects a typical sector that a controller may handle within the scenario and to avoid the source and sink areas where aircraft would interfere artificially due to the scenario design. For the weather-line scenario, two areas were selected between the central hazard and the left and right hazards, respectively. For the roundabout scenario, four areas were selected between the central hazard and each of the corner hazards. For both scenarios, the standard deviation of headings for traffic within each area exhibits similar behavior, so only one plot is presented for the areas highlighted in Fig. 5 and 6. For the roundabout scenario, case (c) where aircraft use adaptability shows much less variability in headings than the other cases, which is expected since the traffic for this case is more organized from the plots of flow pattern in Fig. 6. While for weather-line scenario, the difference in heading variability between the cases is not apparent.
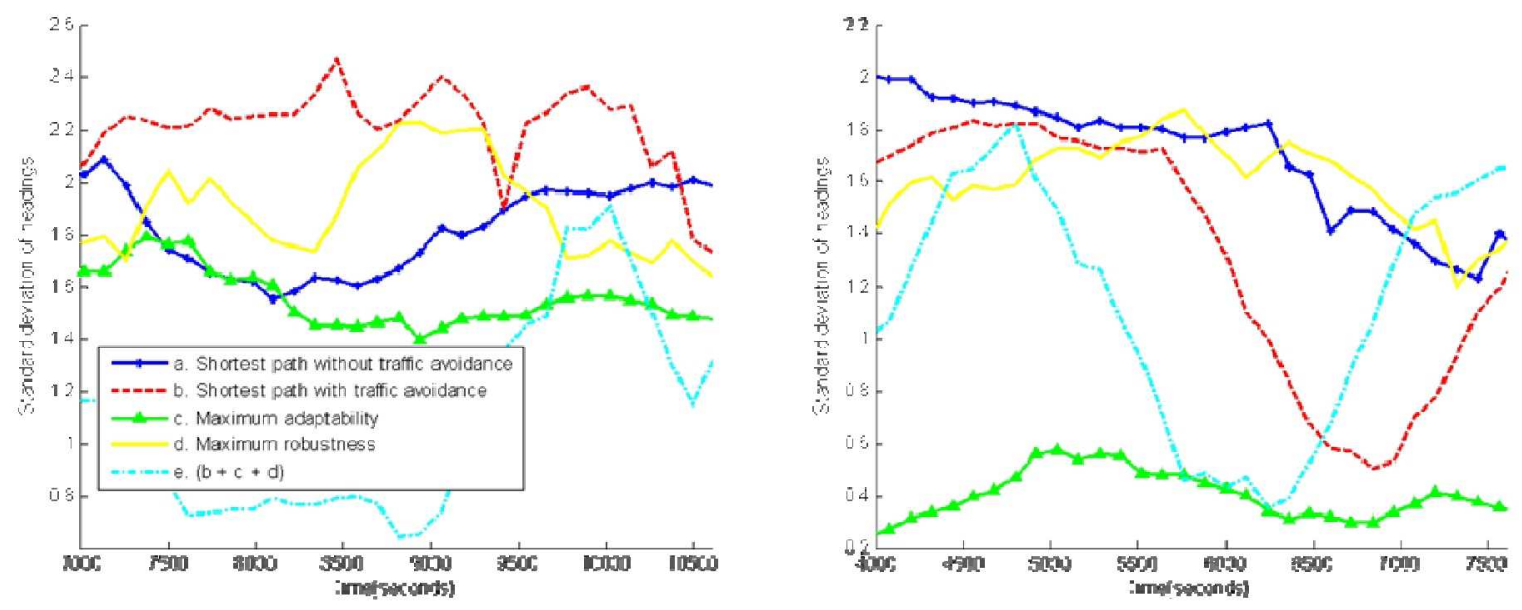

Figure 9. Heading variability analysis

For both scenarios, the total number of the aircraft pairs predicted to be in conflicts in 10 minutes is presented in Fig. 10-a and 10-b using state-based and trajectory-based prediction, respectively. As expected, the case (a) where aircraft used shortest path without traffic avoidance consistently shows the highest number of conflicts. For the twohole scenario of Fig. 5 (left plot), no conflict is detected by for case (c) using maximum adaptability. Meanwhile, the trajectory-based conflicts are much less than state-based conflicts for all the cases, showing that some of the conflicts do not materialize if the traffic intent is accounted for. 

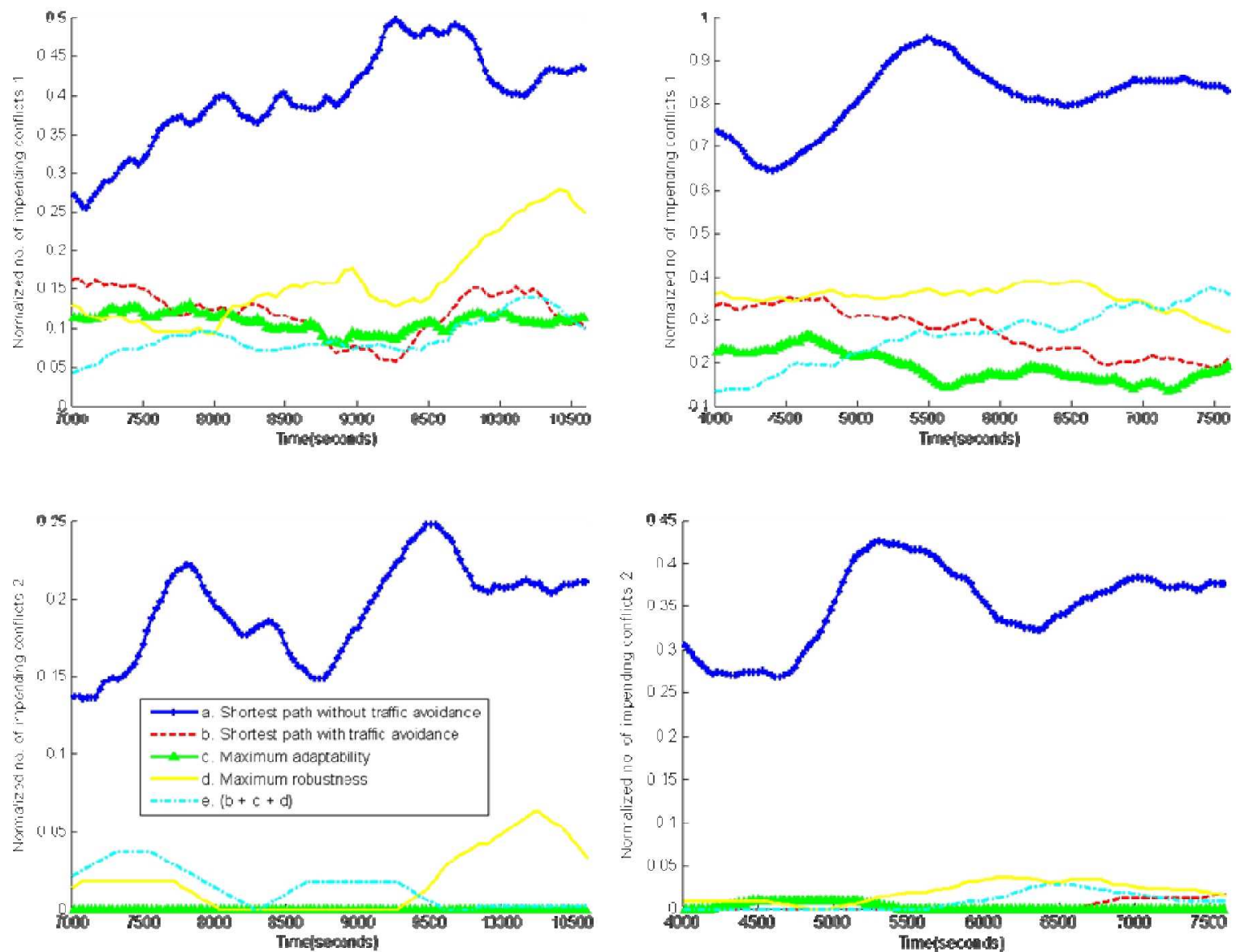

Figure 10. Impending conflicts analysis

It was found from previous plots that case (a) where aircraft use shortest path without traffic avoidance created more impending conflicts than the other four cases. The plots of crossing angle in Fig. 11 show that those conflicts resulting from case (a) are closer to head-on conflicts (180 degree crossing angle). For the roundabout scenario, the crossing angle for case (c) using adaptability is close to 90 degree. A study ${ }^{16}$ mentions that 90 degree conflicts are the least complex. The time-to-conflict metric also showed (plot not included) that case (a) was the worst and case (c) was the best.
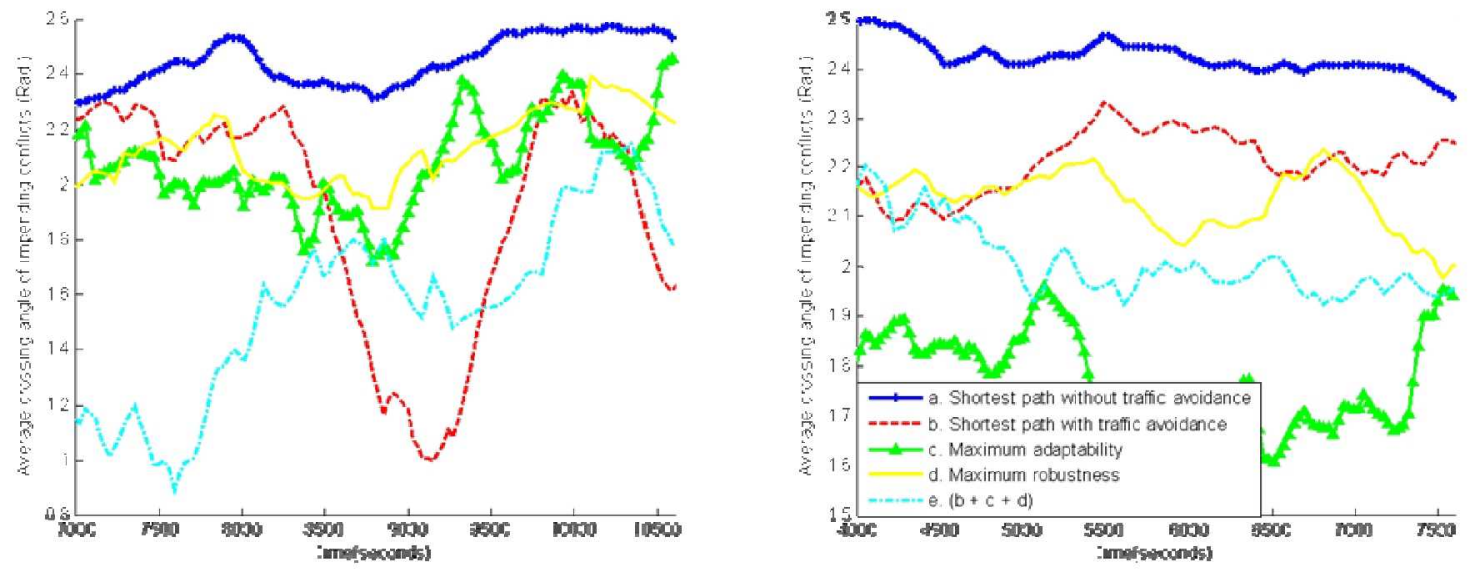

Figure 11. Impending conflicts crossing angle analysis 


\section{Conclusions and Future Extensions}

The analysis reported in this paper demonstrated that using adaptability and robustness metrics in planning flexible aircraft trajectories results in traffic complexity mitigation. Two scenarios showed signs of self separation and self organization when using these metrics. The impact was quantified using dynamic density indicators. They indicated that using adaptability and robustness reduced aircraft density and the potential for conflict between aircraft. These flexibility metrics can be combined with other metrics in the trajectory planning of pilots, airlines, and traffic managers. By incorporating these metrics, the contribution of each aircraft to traffic complexity would be reduced, even without explicit coordination among aircraft or for the aircraft by a ground system.

The results reported in this paper are promising, and open the door for a wide range of future research. Such research extension includes the investigation of: the sensitivity to varying a number of factors such as traffic density and severity of constraints; the effect of dynamic and stochastic decision making where each aircraft updates its trajectory plan over time in response to uncertainty; sensitivity to varying the cost function and the tradeoff between adaptability, robustness and other metrics of interest to users and traffic managers; the effect of non-uniform, competing cost functions among different aircraft; the impact of explicit rules and coordination on furthering self organization; and the application of the metrics and algorithms presented in higher-fidelity real-time systems.

\section{Acknowledgment}

This research was funded by NASA under contract NNA07BA86C.

\section{References}

${ }^{1}$ Joint Planning and Development Office, "Next Generation Air Transportation System Integrated Plan," URL: http://www.jpdo.gov/library/NGATS_v1_1204r.pdf.

${ }^{2}$ Wing, D., "A Potentially Useful Role for Airborne Separation in 4D-Trajectory ATM Operations," Proceedings of the $5^{\text {th }}$ AIAA Aviation Technology Integration and Operations (ATIO) Conference, AIAA-2005-7336, 2005.

${ }^{3}$ Green, S. M., Bilimoria, K. D., and Ballin, M. G., "Distributed Air/Ground Traffic Management for En Route Flight Operations." Air Traffic Control Quarterly, Vol. 9, No. 4, 2001, pp. 259-285.

${ }^{4}$ Idris, H., Vivona, R., Penny, S., Krozel, J., and Bilimoria, K., "Operational Concept for Collaborative Traffic Flow Management based on Field Observations," Proceedings of the $5^{\text {th }}$ ALAA 5th Aviation Technology, Integration and Operations (ATIO) Conference, AIAA-2005-7434, 2005.

${ }^{5}$ Krishnamurthy, K., Barmore, B., and Bussink, F., "Airborne Precision Spacing in Merging terminal Arrival Routes," $\sigma^{\text {th }}$ USA/Europe Air Traffic Management R\&D Seminar, 2005.

${ }^{6}$ Blom H.A.P., B. Klein Obbink, and G.I. Bakker, "Safety Risk Simulation of an Airborne Self Separation Concept of Operation," Proceedings of the $7^{\text {th }}$ AIAA Aviation Technology Integration and Operations (ATIO) Conference, AIAA 2007-7729.

${ }^{7}$ Barhydt, R., and Kopardekar, P., "Joint NASA Ames/Langley Experimental Evaluation of Integrated Air/Ground Operations for En Route Free maneuvering," $6^{\text {th }}$ USA/Europe Air Traffic Management R\&D Seminar, 2005.

${ }^{8}$ Mediterranean Free Flight Programme Final Report, D821, http://www.medff.it/public/index.asp, November 2005.

${ }^{9}$ Erzberger, H., T.J. Davis, and S.M. Green, "Design of Center-TRACON Automation System," AGARD Meeting on Machine Intelligence in ATM, Berlin, Germany, 1993.

${ }^{10}$ Idris H., D. Wing, R. Vivona, and J.L. Garcia-Chico, "A Distributed Trajectory-Oriented Approach to Managing Traffic Complexity," Proceedings of the $7^{\text {th }}$ AIAA Aviation Technology Integration and Operations (ATIO) Conference, AIAA-2007-7731, 2007.

${ }^{11}$ Idris H., R. Vivona, J.L. Garcia-Chico, and D. Wing, "Distributed Traffic Complexity Management by Preserving Trajectory Flexibility," Proceeding of the $26^{\text {th }}$ Digital Avionics Systems Conference, 2007.

${ }^{12}$ Idris H, T. El-Wakil, and D. Wing, "Trajectory planning by preserving flexibility: metrics and analysis," Proceedings of the AIAA Guidance Navigation and Control (GNC) Conference, AIAA-2008-7406, 2008. 
${ }^{13}$ Idris H., and R. Vivona, "Metrics for Traffic Complexity Management in Self-Separation Operations," Air Traffic Control Quarterly, Volume 17, Number 1, 2009.

${ }^{14}$ Laudeman, I.V., Shelden, S.G., Branstrom, R., \& Brasil, C.L., 1999, Dynamic Density: An Air Traffic Management Metric, NASA-TM-1998-112226

${ }^{15}$ Sridhar, B., Sheth, K.S., \& Grabbe, S., Airspace Complexity and its Application in Air Traffic Management, $2^{\text {nd }}$ USA/Europe Air Traffic Management R\&D Seminar, Orlando, Florida

${ }^{16}$ Chatterji, G. B. \& Sridhar B., 2001, Measures for Air Traffic Controller Workload Prediction, Proceedings of the First AIAA Aircraft Technology, Integration, and Operations Forum, Los Angles, CA.

${ }^{17}$ Kopardekar. P. and S. Magyarits, "Measurements and prediction of dynamic density," $5^{\text {th }}$ USA/Europe ATM $R \& D$ Seminar, 2003.

${ }^{18}$ Davison, J., Histon, J., Ragnarsdottir, M., Major, L., Hansman, R.J., "Impact of Operating Context on the Use of Structure in Air Traffic Controller Cognitive Processes". $5^{\text {th }}$ USA/Europe Air Traffic Management R\&D Seminar, 2002.

${ }^{19}$ Delahaye, D. Puechmorel, S., Hansman, R.J., and Histon, J., "Air traffic complexity based on non linear dynamical systems", $5^{\text {th }}$ USA/Europe Air Traffic Management $R \& D$ Seminar, 2003.

${ }^{20}$ Idris H., D. Delahaye, and D. Wing, "Distributed Traffic Flexibility Preservation for Traffic Complexity Mitigation ," $8^{\text {th }}$ USA/Europe Air Traffic Management R\&D Seminar, 2009.

${ }^{21}$ J. Krozel, T. Mueller, and G. Hunter, "Free flight conflict detection and resolution analysis," Proceedings of the AIAA Guidance Navigation and Control (GNC) Conference, San Diego, CA, 1996. 